\title{
A Robust Method for Quantification of IKr-Related T-Wave Morphology Abnormalities
}

\author{
MP Andersen ${ }^{1}$, JQ Xue $^{2}$, C Graff $^{1}$, TB Hardahl ${ }^{1}$, E Toft $^{1,3}$, \\ JK Kanters ${ }^{3,4}$, M Christiansen ${ }^{5}$, HK Jensen ${ }^{6}$, JJ Struijk ${ }^{1}$ \\ ${ }^{1}$ Department of Health Science and Technology, Aalborg University, Aalborg, Denmark \\ ${ }^{2}$ GE Healthcare, Milwaukee, WI, USA \\ ${ }^{3}$ Gentofte and Aalborg University Hospital, Denmark \\ ${ }^{4}$ Danish National Research Foundation Centre for Cardiac Arrhythmia (DARC), University of \\ Copenhagen, Copenhagen, Denmark \\ ${ }^{5}$ Statens Seruminstitut \\ ${ }^{6}$ Aarhus University Hospital Skejby, Aarhus, Denmark
}

\begin{abstract}
The QTc interval plays an important role in premarket testing of new drugs, but the intrinsic variability of the measurement is critical. Most arrhythmogenic drugs inhibit the $I_{K r}$ current and cause both QTc prolongation and changes in T-wave morphology. Quantification of T-wave morphology may be useful in drug testing, but no robust method exists for this purpose. We present a method for quantification of $I_{K r}$-related $T$ wave morphology changes: T-wave asymmetry, flatness and the presence of notches on the T-wave combined to an overall morphology combination score (MCS). In a population of 30 LQT2-subjects (congenital $I_{K r}$ inhibition) and 1096 healthy subjects, both QTcF and MCS yield clear separation between the groups $(p<0.001)$, sensitivity 90\%, specificity 95\%.
\end{abstract}

\section{Introduction}

The QTc interval is the most commonly used measure to quantify repolarization abnormalities. It is a wellknown requirement to measure QTc interval prolongation in "Thorough QT Studies" during the pre-marketing phase of drug evaluation. Still it is widely recognized by the scientific environment, the pharmaceutical industry and the relevant authorities that QTc is associated with limited sensitivity and extensive variability.[1]

Most of the drugs deemed arrhythmogenic and withdrawn from the market have been $\mathrm{I}_{\mathrm{Kr}}$ inhibitors. [1] Canine wedge-model experiments by Yan et Antzelevitch [2] have shown that $\mathrm{I}_{\mathrm{Kr}}$ inhibition is not only expressed by QTc interval prolongation, but also causes transmural dispersion of action potential duration and thus T-wave morphology changes. Several studies have confirmed the phenomenon on the surface ECG in humans.[3,4]
Modest attempts have been made to quantify the repolarization changes by other means than the QTc interval, but so far no robust, widely accepted method has been made available.[1]

Our previous work has shown that measures of $\mathrm{T}$ wave morphology can be used to discriminate between LQT1, LQT2 and healthy subjects.[5] In order to develop a useful alternative to the QTc interval in Thorough QT Studies, we have continued to study T-wave morphology in LQT2 as a biological template of $\mathrm{I}_{\mathrm{Kr}}$ inhibition.

We hypothesize that T-wave morphology parameters describing asymmetry, flatness and notches are potential markers of $\mathrm{I}_{\mathrm{Kr}}$ inhibition and hence discriminate well between healthy subjects and LQT2 patients.

\subsection{Subjects}

Two groups of subjects were included in the study: healthy subjects and a group of LQT2 patients.

The normal group consisted of 1096 healthy subjects, confirmed by history, normal BP and no medication (910 males, 186 females. Age: $10-81 \mathrm{y}$, mean $32.7 \pm 12$ y (SD))

The LQT2 group consisted of 30 genetically confirmed HERG mutation carriers (11 males, 19 females. Age: $19-68$ y, mean $44.5 \pm 14$ y (SD))

The study was approved by the Ethical Committee of the counties Nordjylland and Viborg (no.: vn/2003/129).

\subsection{ECG data collection}

Standard 12-lead ECGs were recorded from all subjects resting in a supine position. Data were recorded with a MAC5000 electrocardiograph (GE Healthcare, Milwaukee, WI) at a sample rate of $500 \mathrm{~Hz}$.

In the normal group one 10 -sec recording was made from each subject. In the LQT2 group five consecutive 10 -sec recordings were made from each subject. 


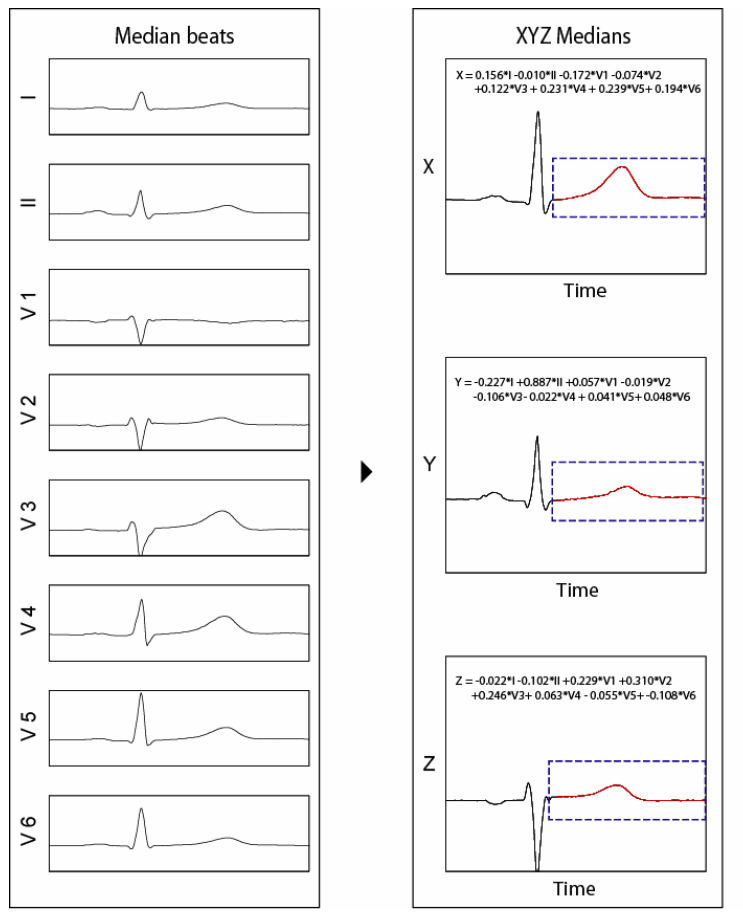

\subsection{ECG preprocessing}

Repeatability of the T-wave morphology quantification depends on the stability of the lead on which morphology is quantified. Earlier studies have shown that V5 is a useful lead for this purpose [5], but it is reasonable to speculate that the optimal lead selection is subject to intra-person variation in anatomy and electrophysiology, electrode placement and variations in signal quality.

To overcome the lead selection problem, a Principal Component Analysis (PCA) was done for each recording. The aim was to obtain a lead that is stable, represents major morphological features of the repolarization phase and reduces intra-person variability.

Each 10 second ECG recording was used to form median beats in all leads using MUSE/Interval Editor software (GE Healthcare, Milwaukee, WI).

Median beats from recorded leads (I-II, V1-V6) were transformed to XYZ leads using a linear transformation to prepare for the following PCA (Figure 1, column 2). In the work published by Xue et al. a novel transformation matrix proved superior to the Inverse Dower transformation with respect to Frank lead resemblance. [6] This Xue transformation was used to create the XYZ leads.

Principal Component Analysis (PCA) was applied to the ST-T segment of XYZ medians to optimize for repolarization information from the ST-T loop. (Figure 1, column 3). PCA was performed using Singular Value Decomposition. The resulting component coefficients

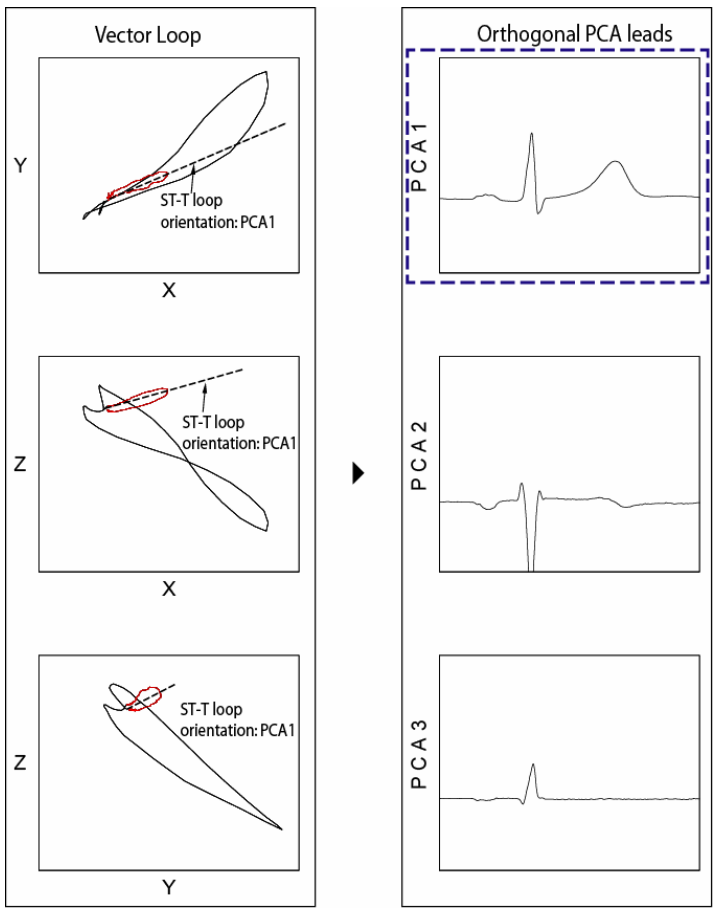

Figure 1. Synthesis of the PCA1 lead obtained by Principal Component Analysis.

(loadings) were used to project the XYZ medians onto the orthogonal eigenvectors describing the spatial direction of the ST-T loop.

This way orthogonal PCA leads optimized for ST-T segment information were formed for the entire median beat as shown in Figure 1, column 4. The T-wave of the first principal component lead (PCA1) derived from XYZ transformed medians can be interpreted geometrically as the optimal projection of the heart dipole vector during the ST-T segment. This lead was therefore used for further analysis of T-wave morphology.

A low-pass Kaiser Window FIR filter with a cutoff frequency of $20 \mathrm{~Hz}$ was applied to the ST-T segment of the PCA1 lead.

Point detection and QT measurements were made automatically using the 12SL algorithm (12SL, GE Healthcare, Milwaukee, WI).

\subsection{T-wave morphology parameters}

T-wave morphology was evaluated in PCA1 by calculation of three morphology features: asymmetry, flatness and notch score.

\section{Asymmetry Score}

The asymmetry score evaluates differences in slope profile and duration of the ascending and descending part of the T-wave. The first derivative of the filtered $\mathrm{T}$ - wave is calculated and divided into two segments 

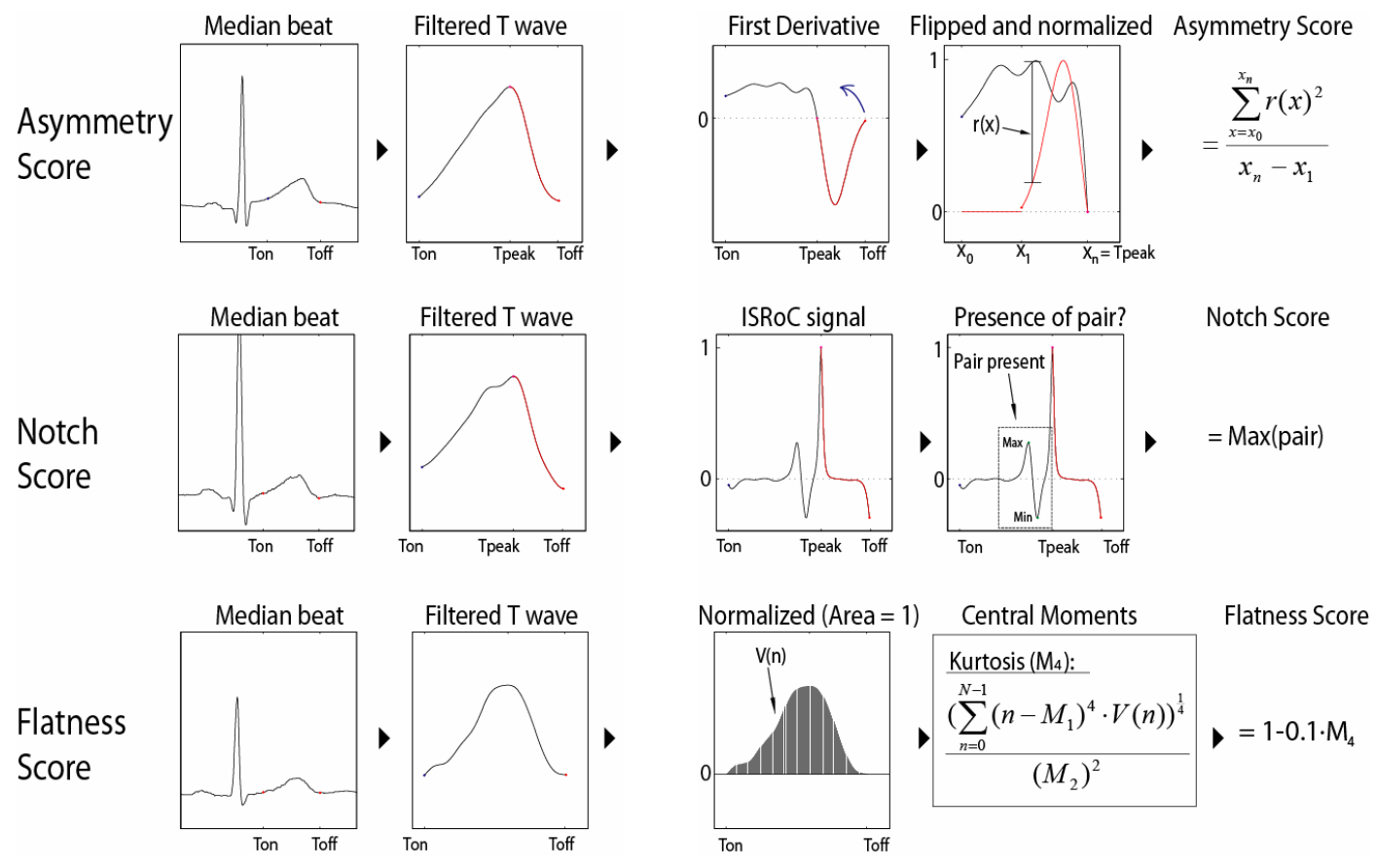

Figure 2. Calculation of T-wave morphology parameters.

corresponding to ascending and descending T-wave. Both segments are normalized with the maximum amplitude of the first derivative within each segment. Then the descending $\mathrm{T}$-wave segment is flipped across the $\mathrm{y}$-axis and $\mathrm{x}$-axis to cover the ascending segment. The segments are compared sample by sample, and the asymmetry score is the average residual between the two segments (Figure 2).

Notch Score

The notch score reflects the size of any visible notches on the T-wave. The score is obtained from the inverse, signed radius of curvature (ISRoC) of the T-wave:

$$
I S R o C=\frac{\frac{\partial^{2} y}{\partial x^{2}}}{\left[1+\left(\frac{\partial y}{\partial x}\right)^{2}\right]^{3 / 2}}
$$

Any deflections on this signal correspond to deviations from the normal, smooth progress of a T-wave. The ISRoC signal is normalized to get a maximum amplitude of 1 . A true notch is reflected as an up-down pair on the ISRoC signal and when such a pair is present, the notch score is defined as the positive peak value of this pair (Figure 2). If no notches are present, the score is 0 .

\section{Flatness Score}

The flatness score is based on kurtosis which is used in statistics to describe the peakedness of a probability distribution. The T-wave amplitude is adjusted to obtain a unit area, and central moments are calculated using these formulae:

$$
\begin{aligned}
& M_{1}=\sum_{n=0}^{N-1} n \cdot V(n) \\
& M_{k}=\left[\sum_{n=0}^{N-1}\left(n-M_{1}\right)^{k} \cdot V(n)\right]^{\frac{1}{k}}
\end{aligned}
$$

where $M_{k}$ is the $k$ 'th central moment, $V(n)$ is the T-wave and $\mathrm{n}$ is the sample number.

Subsequently the fourth central moment $\left(\mathrm{M}_{4}\right)$ is normalized with the variance $\left(\mathrm{M}_{2}\right)$ to obtain a kurtosis measure. The score is then inversed (1-kurtosis) to let increasing values of flatness reflect increasing flatness of the T-wave (Figure 2).

\subsection{Morphology Combination Score}

An overall morphology score was calculated by linear combination of the asymmetry, notch and flatness scores. In our previous study individual features were weighted to obtain the best possible discriminator. [5] In this study, no training was performed to obtain the combination score. Instead the morphology combination score (MCS) was calculated by summing the three morphology scores with equal weights in terms of variance. To obtain equal weights, normalization was done with a robust 99\% estimate of the normal range (NR) of each parameter obtained from the normal group:

$$
\begin{aligned}
\text { MCS } & =\frac{\text { Asymmetry }}{\text { NR(Asymmetry })}+\frac{\text { Notch }}{\text { NR(Notch })}+\frac{\text { Flatness }}{\text { NR(Flatness })} \\
& =1 \cdot \text { Asymmetry }+1.9 \cdot \text { Notch }+1.6 \cdot \text { Flatness }
\end{aligned}
$$



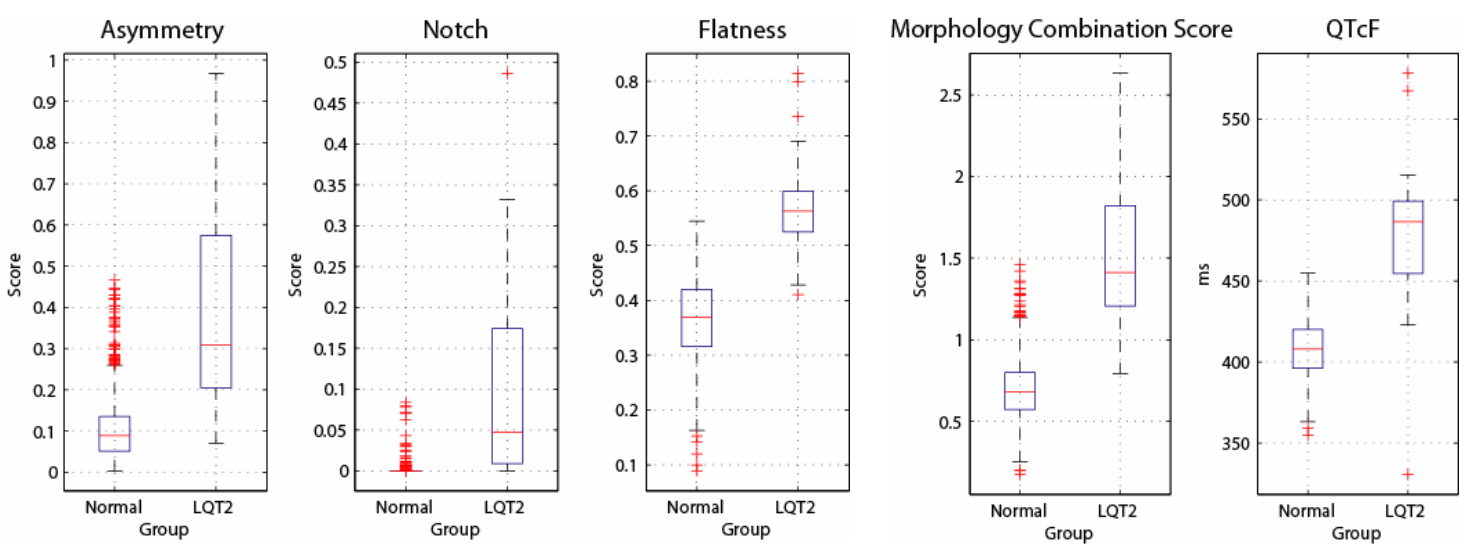

Figure 3. Distribution of T-wave morphology parameters and QTcF in the study population - 1096 normal, 30 LQT2.

\section{Results}

Results of the analysis for lead PCA1 are shown in Figure 3 and summarized in Table 1.

All morphology parameters and QTcF show clear separation between the normal group and the group of LQT2 patients $(\mathrm{p}<0.001)$. Both the Morphology Combination Score (MCS) and QTcF achieve a specificity of $95 \%$ and a sensitivity of $90 \%$. Correlation with QTcF in the normal group was very low for all morphology parameters $\left(R^{2} \leq 0.19\right)$.

\begin{tabular}{|c|c|c|c|}
\hline & Normal $(N=1096)$ & LQT2 $(\mathrm{N}=30)$ & $\mathbf{R}_{(+)}^{2}$ \\
\hline MCS $^{\circ}$ & $0.7 \pm 0.2$ & $1.5 \pm 0.7^{*}$ & 0.12 \\
\hline QTcF $^{\circ}$ & $409 \pm 18$ & $482 \pm 37^{*}$ & 1 \\
\hline Asymmetry $^{\circ}$ & $0.11 \pm 0.10$ & $0.49 \pm 0.65^{*}$ & 0.03 \\
\hline Notch $x$ & $0.000 \pm 0.000$ & $0.043 \pm 0.107 \#$ & 0.01 \\
\hline Flatness $^{\circ}$ & $0.37 \pm 0.08$ & $0.56 \pm 0.09 *$ & 0.19 \\
\hline $\begin{array}{l}{ }^{\circ} \text { Mean } \pm \text { SD } \\
\text { a Median } \pm \text { IQR }\end{array}$ & \multicolumn{3}{|c|}{$\begin{array}{l}* \mathrm{p}<0.001 \text {,Independent Samples T-test } \\
\# \mathrm{p}<0.001 \text {, Mann-Whitney U-test } \\
(+) \text { Correlation coefficient }\left(\mathrm{R}^{2}\right) \text { with } \mathrm{QTcF}\end{array}$} \\
\hline
\end{tabular}

Table 1. Analysis results - normal vs. LQT2, lead PCA1.

An example of the characteristic difference in T-wave morphology between healthy subjects and LQT2 patients is shown in Figure 4.

Figure 4. Unfiltered median beats from lead PCA1. Notice the asymmetric, flat and notched appearance of the LQT2 T-wave.

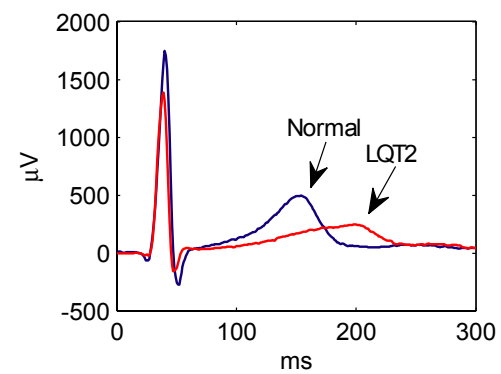

\section{Discussion and conclusions}

The clear separation between healthy subjects and LQT2 patients strongly encourages the use of the Morphology Combination Score for the detection of $\mathrm{I}_{\mathrm{Kr}}$ inhibition. Low correlation with QTcF suggests that the morphology parameters contribute with novel, independent information in the quantification of $\mathrm{I}_{\mathrm{Kr}}$ inhibition. Further work will be done to investigate the usefulness of the method in Thorough QT Studies by investigating $\mathrm{T}$-wave morphology changes induced by known, arrhythmogenic $\mathrm{I}_{\mathrm{Kr}}$ inhibiting drugs such as Sotalol.

\section{References}

[1] Roden DM. Drug-Induced Prolongation of the QT Interval. New England Journal of Medicine. 2004;350(10):1013-22.

[2] Yan GX, Antzelevitch C. Cellular basis for the normal T wave and the electrocardiographic manifestations of the long-QT syndrome. Circulation. 1998;98(18):1928-36.

[3] Kanters JK, Fanoe S, Larsen LA, Bloch Thomsen PE, Toft E, Christiansen M. T wave morphology analysis distinguishes between KvLQT1 and HERG mutations in long QT syndrome. Heart Rhythm. 2004;1(3):285-92.

[4] Couderc JP, McNitt S, Xia J, Zareba W, Moss AJ. Repolarization morphology in adult LQT2 carriers with borderline prolonged QTc interval. Heart Rhythm. 2006;3(12):1460-6.

[5] Struijk JJ, Kanters JK, Andersen MP, Hardahl T, Graff C, Christiansen M, et al. Classification of the long-QT syndrome based on discriminant analysis of T-wave morphology. Medical and Biological Engineering and Computing. 2006;44(7):543-9.

[6] Xue J, Aufderheide T, Scott Wright R, Klein J, Farrell R, Rowlandson I, et al. Added value of new acute coronary syndrome computer algorithm for interpretation of prehospital electrocardiograms. Journal of Electrocardiology. 2004;37(Supplement 1):233-9.

Mads Peter Andersen, mpa@hst.aau.dk Department of Health Science and Technology Aalborg University, Denmark 